\title{
Microspheres an innovative approach in drug delivery system
}

\begin{abstract}
Targeting of active drug moieties to specific body sites with controlled and predetermined drug release from the drug delivery systems have great impact on human health care. Novel drug delivery technology provides an effective approach for entrapment of therapeutically active drugs in multiple unit dosage forms such as microparticles and nanoparticles etc, which transforms the absorption and kinetic properties of the drug molecules. Among multiple unit dosage forms microspheres plays an important role in arena of particulate type of drug delivery systems due to better entrapment, small size with good release characteristics. These systems are coupled with various advantages such as improved therapeutic efficacy with better compliance, encapsulation of variety of drug molecules (macro and micromolecules) low toxicity in comparison to conventional dosage forms. Current manuscript highlights the concept of microspheres with their benefits as drug delivery system and different types of microspheres used for better therapeutic effects.
\end{abstract}

Keywords: novel drug delivery, microspheres, drug release, bioavailability
Volume 5 Issue I - 2018

\author{
Neeta Solanki \\ Department of Pharmaceutical Sciences, Maharshi Dayanand \\ University, India
}

\begin{abstract}
Correspondence: Neeta Solanki, Department of
Pharmaceutical sciences, Maharshi Dayanand University,

Rohtak-I2400 I, Haryana, India, Tel +91-099965 I I834,
\end{abstract}

Email neetasolanki86@gmail.com

Received: December 27, 2017| Published: February 19, 2018

\section{Introduction}

Recently micro particles or microspheres have gained great attention due to their free flowing powder characteristics and biodegradable nature generally made up of protiens or synthetic polymeric materials having particle size in the range of $1-200 \mu \mathrm{m}$. Depending upon the encapsulation of active drug moieties there are two types of microspheres (microcapsules and micro matrices).

Table I Various advantages of microspheres. ${ }^{6-8}$
Microcapsules are multiple unit dosage forms in which active drug molecule is encapsulated and surrounded by separate polymeric wall but in case of micro matrices drug is uniformly dispersed throughout the polymeric matrix. ${ }^{1-3}$ Microspheres have number of advantages over conventional drug delivery systems including an important or effective for administration of therapeutic active molecules in controlled and sustained manner to the target site with improved therapeutic effects ${ }^{4,5}$ (Table 1).

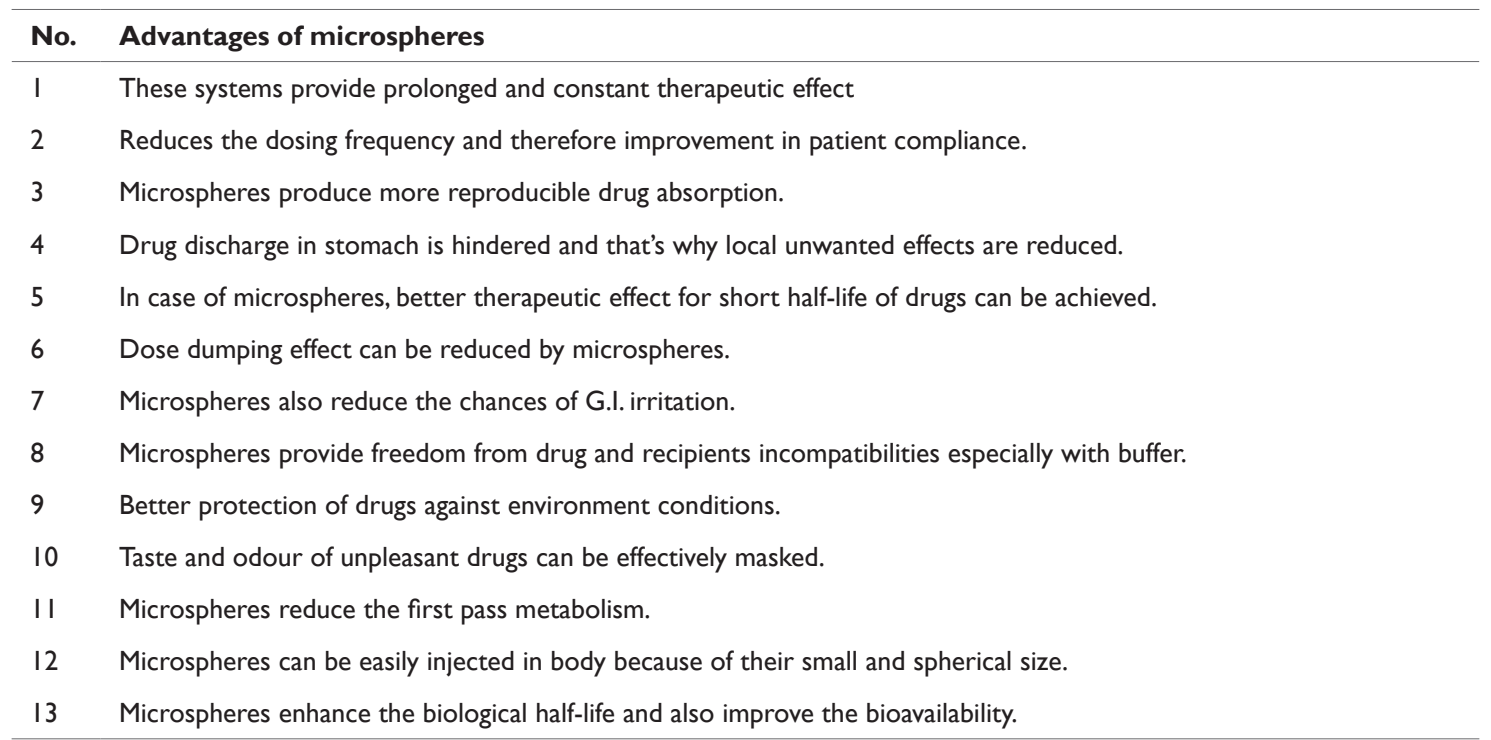

Materials used in the development of microsphere

\section{Natural polymeric materials}

Depending upon the source from which these materials are obtained these are further classified as:

a. Proteins: Collagen, Albumin and Gelatin. b. Carbohydrates: Starch, Agarose, Chitosan and Carrageenan.

c. Chemically modified carbohydrates: Poly dextran, Poly Starch. ${ }^{9,10}$

\section{Synthetic polymers}

These polymeric materials are synthesized by using different chemical substances given below: 
a. Biodegradable polymers include Lactides, Glycolides \& their copolymers, Poly anhydrides and Poly alkyl cyanoacrylates etc.

b. Non-biodegradable polymers includes Poly methyl methacrylate (PMMA), Glycidyl methacrylate, Acrolein, Epoxy polymers. ${ }^{4,11}$

\section{Types of microspheres}

\section{Bioadhesive microspheres}

Bioadhesion term is generally used to for the polymeric materials that have binding ability to the biological membranes (mucous membranes such as buccal, ocular, rectal and nasal etc.). Moreover, adhesion is sticking property of water soluble polymers to

the membrane. Bioadhesive microspheres are (small size and effective drug encapsulation) drug delivery systems involve creation of intimate and prolonged contact of active ingredients with biological tissues and prolongation of contact at target site. Prolongation of residence times further improves the drug absorption effects which help in control over drug release characteristics, reduction of dosing frequency and better patient compliances. ${ }^{12}$

\section{Magnetic microspheres}

An ideal drug delivery system is one which can deliver and localize the active ingredient to the target site particularly in case of cancer disease. Magnetic microspheres are drug delivery systems which can replace large quantity of free circulating by smaller amount of magnetically encapsulated drug. These systems have unique property of showing magnetic behaviour in presence of magnetic field. Different types of proteins and peptides can be targeted by these microspheres in an improved way and magnetic microsphere is an effective approach for drug targeting to tumors for cancer treatment. Basic principle behind the formation of magnetic microspheres is based on the fact that the drug can either encapsulated or conjugated on microsphere surface. Site specific accumulation of active drug molecules to target site which further transfer drug to the local site. ${ }^{13}$

\section{Floating microspheres}

Floating microspheres are low density systems remains buoyant in gastric content for prolonged period of time. Variations in gastric emptying rates of conventional dosage forms can be reduced by these systems due to prolongation of gastric retention time of dosage forms. In addition to this drug is released slowly and constantly at desired rate from the floating microspheres. There is reduction in plasma concentration fluctuations due to low density of microspheres and remain floated in gastric content. These systems also reduce the dose dumping phenomena and provide prolonged and controlled therapeutic effects. ${ }^{2}$

\section{Radioactive microspheres}

Radioactive microspheres are of particular interest in case of cancer treatment because these micro particulates get trapped in first capillary bed of arteries and reach to the tumor of interest. Radioactive microspheres without affecting normal healthy cells deliver radiating dose to the targeted cancerous cell. Radioactivity is liberated by radio isotopes within the microspheres. Various types of radioactive microspheres include different typical distance and the different emitters such as $\alpha$ emitters, $\beta$ emitters, $\gamma$ emitters. ${ }^{14}$

\section{Mucoadhesive microspheres}

Mucoadhesive microspheres are of great pharmaceutical interest due to their adhesive nature to the mucous membrane of nasal cavity, eye, and urinary tract. These systems are well suited for both systemic as well as localized. Mucoadhesive microspheres either consist of entire mucoadhesive polymer or have an outer coating. Better absorption with improved bioavailability of various drugs due to high contact of dosage with mucous membrane and specific drug targeting to the particular site are main advantages which make them an effective drug delivery carrier for variety of drugs. ${ }^{15}$

\section{Polymeric microspheres}

Various types of polymeric materials are used as drug delivery system for pharmaceutical applications which includes.

\section{Biodegradable microspheres}

A wide variety of natural and synthetic polymeric materials are used for this purpose. These polymers have various advantages but important point of concern of these materials is their biocompatibility, biodegradability and bioadhesive property. Gel formation takes place in contact with aqueous body fluids due to swelling behavior of these polymers. Concentration of polymer directly affects the rate and extent of drug release. Drug is released from the microspheres in predetermined and controlled manner. Main constraints associated with these polymeric materials are not control to develop microspheres with good loading capacity and restricts their clinical use. ${ }^{16}$

\section{Conclusion}

Microspheres have been appeared as effective controlled release dosage forms and represents great pharmaceutical applications in area of drug delivery technology with multidisciplinary advancements for treatment of number of diseases. Microspheres because of their attractive properties in terms of patient compliance, therapeutic efficacy, and reduction in side effects these delivery systems provide various therapeutic benefits over conventional dosage forms. There is further place for improvement in future in microsphere drug delivery systems for more therapeutic results. Combination of microparticles with different novel strategies particularly in diagnostic area, diseased cell sorting, entrapment of genetic materials and tissue engineered products within the matrix.

\section{Acknowledgements}

None.

\section{Conflict of interest}

The author declares no conflict of interest.

\section{References}

1. Alagusundaram M, Chetty MSC, Umashankari K, et al. Microspheres as a novel drug delivery sysytem - a review. Int $J$ ChemTech Res. 2009;1(3):526-534

2. Kadam NR, Suvarna V. Microspheres: a brief review. Asian J Biomed Pharma Sci. 2015;5(47):13-19.

3. Sailaja KA, Anusha K, Jyothika M. Biomedical Applications of Microspheres. J modern drug discov Drug delivers. 2016;4(2):1-5.

4. Ramteke KH, Jadhav VB, Dhole SN. Microspheres: as carriers used for novel drug delivery system. J Pharm. 2012;2(4):44-48. 
5. Kataria S, Middha A, Sandhu P, et al. Microsphere: A Review. Int J Res Pharm Chem. 2011;1(4):1184-1198.

6. Sharma N, Purwar N, Gupta PC. Microspheres as drug carriers for controlled drug delivery: a review. Int J Pharma Sci Res. 2015;6(11):45794587.

7. Nair N, Reddy BH, Kumar CKA, et al. Application of chitosan microspheres as drug carriers: a review. J Pharm Sci Res. 2009;1(2):1-12.

8. Nikam VK, Gudsoorkar VR, Hiremath SN, et al. Microspheres - a novel drug delivery system: an overview. Int Pharm Chem Sci. 2012;1(1):113128.

9. Patel NR, Patel DA, Bharadia PD, et al. Microsphere as a novel drug delivery. Int J Pharma Life Sci. 2011;2(8):992-997.

10. Bansal H, Kaur SP, Gupta AK. Microsphere: methods of preparation and applications; a comparative study. Int J Pharmaceutical Sciences Review and Research. 2011;10(1):69-78.
11. Kumar BP, Chandiran IS, Bhavya B, et al. Microparticulate drug delivery system: a review. Indian J Pharma Sci Res. 2011;1(1):19-37.

12. Rajput S, Agrawal P, Pathak A, et al. A review on microspheres: methods of preparation and evaluation. World J Pharm Pharma Sci. 2015;1(1):422-438.

13. Parida KA, Panda SK, Ravanan P, et al. Microparticles based drug delivery systems: preparation and application in cancer therapeutics. Int Arch Appl Sci Technol. 2013;4(3):68-75.

14. Krishna KVM, Reddy CS, Srikanth S. A review on microsphere for novel drug delivery system. Int J Res Pharm Chem. 2013;3(4):763-767.

15. Midha K, Nagpal M, Arora S. Microspheres: a recent update. Int J Recent Sci Res. 2015;6(8):5859-5867.

16. Rathore B, Yadav A, Nayak G, et al. A review on microspheres as drug delivery carriers for management of diabetes mellitus. Int J Pharm Life Sci. 2012;3(10):2064-2070. 